\title{
Psychiatric emergency service users at Groote Schuur Hospital, Cape Town
}

Don A B Wilson, BSC, MB ChB, FCPsych (SA)

Alan J Flisher, MSC (Clin Psychol), MMed (Psych), MPhil (Child and Adolescent Psychiatry), PhD, FCPsych (SA), $\mathrm{DCH}$

Mark Welman, MA, PhD

Department of Psychiatry and Mental Health,

University of Cape Town

Objective. To document and compare the characteristics of patients assessed at a psychiatric emergency service (PES) during April and May of 1988 and 1998.

Design. Two cross-sectional surveys.

Setting. Groote Schuur Hospital (GSH), Cape Town.

Subjects. Patients assessed at the PES, GSH.

Outcome measures. These were occupational status, referral source, distance travelled to get to the hospital, and diagnosis.

Results. Nine hundred and twenty-five patients made 1081 visits to the unit during a 2 -month period in 1988, while during a similar period in 1998, 364 patients made 477 visits. In the latter period the patients were significantly more likely to be skilled workers or students/scholars, to be referred from within GSH or other health facilities, to have travelled less than $10 \mathrm{~km}$ to get to the hospital, and to be suffering from a mood disorder or suicidality land less likely to be suffering from a substance use disorder).

Conclusions. The differences between the two time periods indicate that changes in policy during the period 1988 1998 may have impacted on the patient profile at the PES at GSH. It is important to document such changes with a view to informing service planning for both tertiary referral centres and other levels of care.
A number of intersecting policy developments in recent years may have impacted on the characteristics of patients receiving psychiatric services at tertiary hospitals. For example, there has been increased emphasis on providing mental health care at primary and secondary levels in cases where tertiary-level services are not clinically indicated, and on the integration of mental health services into general health services.' Many of these developments were particularly evident in the years around 1994 when the health policies of the African National Congress replaced those of the last apartheid government. ${ }^{2}$

The aim of this study was to document and compare the characteristics of patients assessed at the psychiatric emergency service (PES) at Groote Schuur Hospital (GSH) in Cape Town in 1988 and 1998. Specifically, we focused on characteristics that may have been affected by the policy developments mentioned above, namely number of patients, occupational profile, diagnosis, referral source, and distance travelled to reach the hospital.

\section{Methods}

In 1988 the PES at GSH provided a service to a catchment area of approximately 2 million people. The staff consisted of a part-ime psychiatrist, 2 psychiatric registrars, a social worker, a trainee clinical psychologist and 7 nurses. In 1998, the staff consisted of a part-time psychiatrist, 3 psychiatric registrars and 7 nurses. After hours, a pool of psychiatric registrars provided medical services on a rotating basis. There were 10 beds in the unit.

The primary role of the PES was to provide prompt and comprehensive assessments and to institute management plans for patients presenting with psychiatric emergencies. Other functions included providing a consultation service to colleagues in the medical emergency services and elsewhere at GSH, providing telephone consultations, providing emergency outpatient consultations for existing psychiatric patients at GSH whether still on treatment or not, providing teaching for medical and other health science students, and conducting research. 
The sample comprised all patients presenting to the PES at GSH during April and May of 1988 and 1998. The registrars who initially assessed the patients completed a questionnaire for each patient recording demographic and clinical data. Diagnoses were based on the 3rd (revised) and 4th editions of the Diagnostic and Statistical Manual of Mental Disorders, ${ }^{3,4}$ in 1988 and 1998 respectively. Collateral information was obtained from escorts such as, relatives, friends and ambulance personnel. Questionnaires were checked the following day and any omissions were rectified after consulting the case notes and/or the registrar who assessed the patient. Differences between the two data collection periods were assessed using chi-squared tests.

\section{Results}

In April and May of 1988, 925 patients made 1081 visits to the unit, while in April and May of 1998, 364 patients made 477 visits. The analyses below are all based on patient visits. The mean patient ages were 33 (range 12 - 83) and 32 (range $11-82$ ) years in 1988 and 1998 respectively. Of the visits in 1988 and 1998, $573(53.0 \%)$ and 303 (63.5\%) respectively were by female patients $\left(\chi^{2}=14.67, p=0.000\right)$.
The occupational profile of the sample is shown in Table I. The majority of patients in both time periods were unemployed or unskilled/casual workers. The proportion of skilled workers and students/scholars increased significantly between 1988 and 1998, whereas the proportion of people receiving pensions or disability grants decreased significantly.

Primary diagnoses are given in Table II. Patients assessed in the later period were more likely to receive diagnoses of depressive or bipolar disorder or suicide attempt, and less likely to receive a diagnosis of substance use disorder (including alcohol withdrawal). The term 'attempted suicide' covered any form of suicidal behaviour that involved a self-inflicted, injurious, sublethal suicide act. Attempted suicide was the primary diagnosis for $108(10.7 \%)$ and 90 (18.8\%) patient visits in 1988 and 1998 respectively (Table II). In addition, attempted suicide was an associated or secondary diagnosis for a further $78(7.7 \%)$ and 20 (4.2\%) patient visits in 1988 and 1998 respectively. In other words, attempted suicide was a prominent feature in 186 $(18.4 \%)$ and $110(23.1 \%)$ visits in 1988 and 1998 respectively, which is a significant difference $\left(\chi^{2}=4.43, p=\right.$ 0.0351 .

\section{Table I. Occupations of patients attending the psychiatric emergency services unit, Groote Schuur Hospital, 1988 and $1998^{*}$}

\begin{tabular}{|c|c|c|c|c|c|c|}
\hline \multirow[b]{2}{*}{ Occupation } & \multicolumn{2}{|c|}{$1988(N=975)$} & \multicolumn{2}{|c|}{$1998(N=468)$} & \multirow[b]{2}{*}{$x^{2}$} & \multirow[b]{2}{*}{$\mathrm{p}$} \\
\hline & $N$ & $\%$ & $N$ & $\%$ & & \\
\hline Unemployed & 366 & 37.5 & 171 & 36.5 & 0.14 & 0.713 \\
\hline Unskilled/casual & 176 & 18.1 & 75 & 16.0 & 0.90 & 0.342 \\
\hline Skilled (e.g. police, artisan, clerical) & 117 & 12.0 & 93 & 19.9 & 15.76 & $0.000^{\ddagger}$ \\
\hline Pensioner or on disability grant & 117 & 12.0 & 29 & 6.2 & 11.71 & $0.001^{\ddagger}$ \\
\hline Student/scholar & 97 & 9.9 & 65 & 13.9 & 4.93 & $0.026^{\dagger}$ \\
\hline Housewife & 64 & 6.6 & 24 & 5.1 & 1.14 & 0.286 \\
\hline Professional or managerial & 38 & 3.9 & 14 & 3.0 & 0.75 & 0.387 \\
\hline
\end{tabular}

Table II. Primary diagnosis of patients attending the psychiatric emergency services unit, Groote Schuur Hospital, 1988 and $1998^{*}$

\begin{tabular}{|c|c|c|c|c|c|c|}
\hline \multirow[b]{2}{*}{ Disorder } & \multicolumn{2}{|c|}{$1988(N=1009)$} & \multicolumn{2}{|c|}{$1998(N=476)$} & \multirow[b]{2}{*}{$x^{2}$} & \multirow[b]{2}{*}{$p$} \\
\hline & $N$ & $\%$ & $N$ & $\%$ & & \\
\hline Depressive and bipolar disorder & 249 & 24.7 & 142 & 29.7 & 4.43 & $0.035^{\dagger}$ \\
\hline Substance use disorder (including & & & & & & \\
\hline alcohol withdrawal) & 233 & 23.1 & 63 & 13.2 & 19.69 & $0.000^{\ddagger}$ \\
\hline Schizophrenia and other psychoses & 168 & 16.7 & 61 & 12.8 & 3.65 & 0.056 \\
\hline Suicide attempt & 108 & 10.7 & 90 & 18.8 & 18.84 & $0.000^{\ddagger}$ \\
\hline Anxiety disorder & 57 & 5.6 & 18 & 3.8 & 2.35 & 0.125 \\
\hline Adjustment disorder & 52 & 5.2 & 30 & 6.3 & 0.82 & 0.366 \\
\hline Other disorder & 68 & 6.7 & 38 & 7.9 & 0.75 & 0.384 \\
\hline No psychiatric disorder & 20 & 2.0 & 8 & 1.7 & 0.16 & 0.690 \\
\hline
\end{tabular}


In 1988 patients were significantly more likely to be seen without referral (in other words first appointments without referral or unscheduled follow-up visits) than in 1998 (993, 91.9\% v. $313,65.6 \%)\left(\chi^{2}=168.08, p=0.000\right)$.

Similarly, patients in 1988 were less likely to be referred either from within $\mathrm{GSH}\left(208,19.2 \%\right.$ v. 141, 29.6\%) $\left(\chi^{2}=20.27\right.$, $p=0.000)$ or from other health facilities, including from general practitioners $\left(359,33.2 \%\right.$ v. 208, 43.6\%) $\left(\chi^{2}=\right.$ 15.45, $p=0.0001$.

The proportion of patients who travelled more than $10 \mathrm{~km}$ to the hospital was higher in 1988 (653,60.4\%) than in 1998 (241, $50.5 \%)\left(\chi^{2}=13.22, p=0.000\right)$.

\section{Discussion}

The number of patients seen at the PES at GSH decreased substantially between 1988 and 1998, with the number seen in 1998 constituting $39.4 \%$ of that seen in 1988 . The study did not provide data to elucidate the question of whether patients who would have received services at the GSH PES in 1988 received such services elsewhere, and if they did, where they received such services. It is possible that many of these patients received services at primary and secondary levels of care. If this was the case it would indicate that the policy of providing services at 'lower' levels of service where appropriate had been successful, resulting in considerable financial benefits. They may also have received services at other mental health facilities initiated in the decade under consideration, for example in the private or non-governmental sectors. The quality and quantity of psychiatric education provided for medical students at the University of Cape Town improved markedly between 1988 and 1998. It is possible that recent graduates working at primary and secondary levels were more likely to manage patients experiencing psychiatric emergencies themselves as opposed to referring them to a tertiary referral centre. Finally, in the decade under consideration there was an increase in the availability of other mental health services that may have been used, particularly in primary health care settings and nongovernmental organisations.

In both time periods the majority of patients were unemployed or unskilled/casual workers, which indicates that the service addressed the needs of the most disadvantaged members of society. The increased proportion of skilled workers and students/scholars in the later time period may reflect the fact that it was more difficult to secure an appointment in 1998 than in 1988. The requirement that there should be a referral letter from a medical practitioner working at primary or secondary level was enforced more consistently in the later time period. Therefore those with greater access to resources (such as skilled workers or students) may have been more likely to be able to secure such a referral letter. The converse argument could explain the finding that there was a significantly smaller proportion of people receiving pensions or disability grants in the later time period. They may have been less likely to secure a referral letter. In addition, they may be less likely to afford the costs of attending a tertiary referral hospital.

There was a significant reduction in the proportion of patients with a diagnosis of substance use disorder from 1988 to 1998. This was probably largely due to a change of hospital policy. In 1988 patients were routinely managed at the PES at GSH. However, in the period between 1988 and 1998 it was agreed that a subset of patients would be managed in medical wards at GSH, thus reducing the demand for alcohol withdrawal services at the PES. Also, patients were increasingly managed at primary and secondary-level facilities. Furthermore, before 1998 GSH had closed its inpatient addiction unit, and without inpatient facilities patients were no longer using the emergency facilities for detoxification.

Conversely, there was a higher proportion of patients with depressive and bipolar disorder in 1998 compared with 1988. This finding could be explained by an increase in the prevalence of depression in the community from which GSH draws its patients. Community-based cohort or sequential crosssectional studies would be necessary to assess whether any specific trends are present. However, there are no a priori reasons to suspect that the prevalence of mood disorders would increase so substantially over such a short time period. An alternative and more plausible explanation is that there was an increased level of skill in the detection of mood disorder. There was an increase in the proportion of attempted suicide patients, both when considering attempted suicide as a diagnosis and when considering it in patients for whom alternative diagnoses were entered. This increase could be a manifestation of the increase in the proportion of patients with mood disorders, with which suicide attempts are frequently associated. An alternative explanation is that there has been an increase in the rate of attempted and completed suicide. There are no South African data addressing this possibility directly. However, an analysis based on nationally registered South African suicide mortality 
data found that there was an increase in the suicide rate for young white males and elderly white males and females from 1968 to 1990.5 Finally, health system factors could have contributed to the increase in the proportion of patients assessed after attempting suicide. Specifically, patients may have been more likely to be referred from primary and secondary levels because of shortages of social workers at these levels and concerns about whether the assessment was adequate (including the prospect of litigation).

Patients were more likely to be referred either from within GSH or other health facilities in 1998 than in 1988. This reflects a positive development in that it is possible that greater numbers of patients are being assessed and treated outside GSH. Indeed, in a large number of cases it is preferable that patients be assessed and treated at primary and secondary levels, or in the private sector, partly for reasons of cost effectiveness. The fact that a significantly lower proportion of patients travelled less than $10 \mathrm{~km}$ to get to GSH in 1998 than in 1988 is consistent with the possibility that a relatively greater number of patients were receiving mental health services outside GSH.

\section{Conclusion}

A number of findings indicate that changes in policy during the period 1988 - 1998 may have impacted on the patient profile at the PES at GSH. In the latter period there were fewer patients, and they were more likely: (i) to be skilled workers or students/scholars; (ii) to be referred from within GSH or other health facilities; (iii) to have travelled less than $10 \mathrm{~km}$ to get to the hospital; and (iv) to be suffering from a mood disorder or suicidality land less likely to be suffering from a substance use disorder). The findings suggest that changes in mental health policy can have substantial and immediate effects on the patient profile in a tertiary referral hospital. It is important to document such changes with a view to informing service planning both for tertiary referral centres and other levels of care.

\section{References}

1. Flisher AJ, Subedar H, Fisher W. Mental heal h. In: Crisp N, Nuli A, eds. South African Health Review 1999. Durban: Health Systems Trust, 1999

2. Department of Heal h. White Paper for the Transformation of the Health System in South Africa (Government Gazette No. 17910). Pretoria: Government Printer, 1997.

3. American Psychiatric Association. Diagnostic and Statistical Manual of Mental Disorders. 3rd ed, revised. Washington, DC: American Psychiatric Association, 1987.

4. American Psychiatric Association. Diagnostic and Statistical Manual of Mental Disorders. 4th ed. Washington, DC: American Psychiatric Association, 1994.

5. Flisher AJ, Liang H, Laubscher R, Lombard C. Suicide trends in South Africa 1968 1979. Scand J Public Health 2004; 32: 411 1-418. 